\title{
Debate DEBATE \\ View 1: Assisted conception in couples with HIV infection
}

\author{
S Sharma, C Gilling-Smith, A E Semprini, S E Barton, J R Smith
}

This review aims to address the need to alter our current clinical practice to meet the reproductive desires of the HIV in fected couples.

\section{S Sharma}

C Gilling-Smith,

J R Smith Department of

Gynaecology, Chelsea and

Westminster Hospital,

London, UK

A E Semprini Department of Obstetrics, Milano

University Hospital, Milan,

Italy

S E Barton Department of Genitourinary/HIV

Medicine, Chelsea and

Westminster Hospital,

London, UK

Correspondence to: Ms C Gilling Smith, Assisted Conception Unit Chelsea and Westminster Hospital, 369 Fulham Road, London SW10 9NH, UK; cgs@chelwest.nhs.uk

Accepted for publication 29 November 2002
Infertility centres are increasingly dealing with couples with HIV who strongly want to be parents. ${ }^{1}$ Preconceptional counselling and reproductive assistance for such couples have significant ethical and practical implications for the couple and the carers. This article addresses the ethical and practical issues concerned in assisting them to limit negative consequences, both for the couples and the future child.

In general, there are four underlying principles that constitute the framework of medical ethics:

(I) Primum non nocere: first of all, do no harm

(ii) Beneficence: do good

(iii) Autonomy: respect the individual

(iv) Justice: honest and fair care.

Thus, these principles of medical ethics stress the need to do as much good as possible while keeping the harm/risks of any intervention to the minimum. To respect the patient's autonomy, they need to be informed of the diagnosis and management options (pros and cons) in terms they understand and be allowed to make choices for their care without undue pressures from carers. Finally, the treatment must be administered in a just and fair manner. ${ }^{2}$

We present three clinical scenarios of couples demonstrating the dilemmas faced in the management of these situations.

(1) The man is infected with HIV and the woman is not

(2) The woman is infected with HIV and the man is not

(3) Both the man and the woman are infected

When these couples are fertile (for example, they have conceived spontaneously in the past) medical intervention aims at reducing the risk of viral transfer to the uninfected partner and the future child. This does not pose a substantial ethical problem, although those against these couples conceiving can argue that providing preconception counselling may encourage them to conceive, thus posing a risk for the uninfected partner and/or the child and that this risk would not be present otherwise.

In couples who have already tried to conceive unsuccessfully, medical intervention permits the conception of a child who is at risk of acquiring HIV, even with optimal reproductive care. It can be debated whether the couple's desire to have a child justifies medical intervention that involves the potential risk of infection for the healthy partner and/or the child.

When only the man is infected with HIV, semen washing and reproductive technology can reduce and possibly eliminate the risk of infection for both the woman and the conceptus. When only the woman is infected, insemination with the partner's semen eliminates the risk of infection for the man, but the infant will be exposed to the risk of vertical acquisition of HIV from the infected mother. When both partners are infected, one partner having infected the other and both are antiretroviral treatment naive, they may conceive on their own, as there is no reproductive technology that may reduce the risk of infection for the child. When the man and the woman were infected at different times or from different sources, the viral mutations may vary according to the length of exposure to specific antiretroviral drugs. In this situation, semen washing reduces the possibility of transmission of mutated virus through unprotected penetrative intercourse, which may create a double infection in the partners.

Preconception counselling should help the woman to modify or initiate antiretroviral treatment to reduce both embryo-fetal toxicity and risk of vertical transmission. ${ }^{4}$

To illustrate these scenarios, we present each as a case history.

\section{SCENARIO}

In a couple where the man is infected with HIV and the woman is not, she is at risk of contracting the infection if she tries to achieve fertilisation through unprotected intercourse.

\section{Case history}

A 37 years old man living in a rehabilitation community for 7 years asked for reproductive assistance to have a child. He had acquired HIV infection through drug use but his wife was uninfected and healthy. They had been married for 5 years and, apart from a brief period at the start of their relationship, they had regularly used condoms to reduce the risk of sexual transmission of HIV. With his current antiretroviral regimen, he had undetectable viraemia and was very tempted to try for a pregnancy by empirically timed unprotected intercourse. The genitourinary medicine specialist had discouraged him from spontaneous attempts at conception and referred him to us for counselling. During the first meeting the couple were informed that $10 \%$ of males with undetectable viral load in blood plasma, have detectable cell associated and cell free virus in their semen and that the chances of pregnancy per episode of unprotected penetrative sex is approximately 15\%, when no infertility factors are present. They were told that there are no data on the transmission risk in this setting and that usually a number of attempts are required to achieve fertilisation and therefore the cumulative risk of infection can be significant. They were 


\section{Debate}

advised to undergo tests to ascertain a normal fertility potential and offered reproductive assistance through semen washing, coupled with intrauterine insemination or extracorporeal fertilisation. The couple had to be treated for chlamydial infection and the man required prolonged antibacterial treatment for prostatitis, which was responsible for reduced semen motility. The woman conceived on the third cycle of insemination with washed semen and no fertility drugs were required as her hormonal profile was optimal. She was seronegative during her 3 monthly serological screening until delivery and the child was born uninfected and healthy.

\section{Discussion}

Transmission of HIV may follow a single penetrative intercourse, the risk being approximately 1:500-1:1000 per sexual encounter. ${ }^{56}$ Thus, the advice to try spontaneously during the fertile period for a pregnancy in these HIV discordant couples carries a risk, which is proportional to the number of attempts, although the pattern of transmission is not linear. The total risk of infection may be increased further by the additional unprotected acts of recreational sex, as the couple may perceive that they are at low risk of sexual transmission.

In 1997, Mandelbrot et al reported the outcome of 104 consecutive pregnancies in 92 HIV negative women with HIV positive partners. All pregnancies were natural conceptions, occurring after basic infertility screening was carried out. The couples were taught to identify signs of ovulation to minimise chances of viral transmission. There were 92 pregnancies, four abortions, six miscarriages, and two were lost to follow up in the second trimester. In this group, there were two seroconversions at 7 months of pregnancy and two postpartum - that is, the risk of seroconversion was $4 / 92(4.3 \%) .^{7}$ This study predates the use of triple antiretroviral therapy and thus the infected males included were unlikely to have had undetectable viral load in their plasma and semen.

In contrast, Semprini et al in Milan have been providing assisted conception to serodiscordant couples through timed insemination attempts after seminal processing to free spermatozoa from HIV containing seminal plasma and seminal leucocytes. ${ }^{89}$ To date, nearly 3000 cycles of intrauterine insemination (IUI) or extracorporeal fertilisation have been completed without any case of HIV infection to the woman or to the children born through this method.

Seminal processing of ejaculates from HIV positive males is carried out through a three step method. The gradient centrifugation step, which traps the seminal leucocytes, is followed by washing of the recovered spermatozoa to eliminate traces of HIV rich seminal plasma and the spontaneous migration step which separates motile sperms from immobile seminal leucocytes that may have filtered through the first two processing steps. The success of this method has been supported by flow cytometric analysis, which has shown that HIV receptors (CD4, CCR5, and CXCR4) are absent on the sperm surface. ${ }^{10}$

In addition to Semprini's centre in Milan, there are other European collaborating centres in
Barcelona, Spain; Chelsea and Westminster Hospital, London and in Birmingham, United Kingdom; St Gallen, Switzerland; Mannheim, Germany, and Toulouse, France, which are providing reproductive assistance to HIV discordant couples. Intrauterine transfer of washed semen is not indicated in HIV discordant couples with untreatable infertility problems impeding in vivo conception. These couples should be helped with extracorporeal fertilisation techniques similar to those who fail to conceive after repeated IUI attempts. In some instances poor seminal quality requires direct gamete manipulation with injection of the washed spermatozoa directly into the egg (ICSI, intracytoplasmic sperm injection) to achieve fertilisation. ${ }^{11}{ }^{12}$ A small number of ICSI attempts have been reported in HIV discordant couples, when compared to the number of IUI and standard in vitro fertilisation (IVF) trials. ${ }^{13}{ }^{14}$ No case of female or infant infection has been reported after ICSI, but couples undertaking ICSI should be informed that safety is based on a limited number of trials. The possibility that free virus can be attached to the external acrosomal membrane, which is eliminated during spontaneous egg penetration but not with ICSI, might potentially render this method riskier than IUI or IVF. Based on this consideration, IUI and IVF should be the first option of reproductive technology in HIV discordant couples who do not have a clinical indication for ICSI.

\section{SCENARIO 2}

In the second scenario, a 26 year old HIV positive woman presented to the assisted conception unit with her HIV negative husband to discuss the implications of pregnancy. Her CD4 count was $0.72 \times 10^{9} / 1$ and her HIV RNA plasma load was below 50 copies $/ \mathrm{ml}$. She had been using condoms regularly to avoid conception and protect her partner from sexual transmission. Following extensive counselling spread over weeks, the couple decided to go ahead with a pregnancy. The woman was helped to make a decision on whether to stop medication during the first trimester, advised on the benefit of pharmacological prophylaxis of vertical transmission coupled with elective abdominal birth and avoidance of breast feeding. They were taught how to perform artificial insemination by the husband's sperm. The husband produced a semen sample into a pot and then, using a $5 \mathrm{ml}$ syringe and a quill, aspirated the seminal fluid. He then injected the semen high into the vagina. They were advised to keep a temperature chart or to buy an ovulation kit to determine when ovulation was occurring. They were advised to repeat this procedure over six cycles with pregnancy being obtained in three. At other times they were recommended to engage only in condom protected penetrative sex.

\section{Discussion}

This scheme of reproductive counselling and at-home reproductive assistance eliminates the risk of infection for the man. Potentially, also the conception delay may be shortened as insemination attempts are timed to ovulation. Genital infections should be excluded in the man as they can be transmitted with the insemination attempts, while assessment of tubal patency may be 
unnecessary if conception occurs within 6 months, as the procedure carries no risk of infection for the uninfected partner.

The woman on antiretroviral treatment should receive information on the effect of discontinuing therapy in the first trimester to avoid potential teratogenesis. At present there are no data to support or refute this. Her immunological and virological situation should be compatible with such a break in treatment and she must be informed that its effect on viral resistance is unclear at present. Asymptomatic women with low viral loads and good CD4 counts do not need antiretroviral therapy and should be managed the same in the first trimester as in the non-pregnant state. $^{415}$ Providing this information and basic reproductive care in this setting could be perceived as unjustified since the medical intervention is putting a child-to-be at risk of acquiring HIV. This objection is obviously focused only on the clinician's duty towards the patient, while significantly less attention is given to the reproductive desires and rights of women with HIV. The demonstrated efficacy of interventions to reduce vertical transmission ( $2 \%$ average between studies $v 21 \%$ before introduction of vertical transmission preventive measures $)^{16}{ }^{17}$ seems now to have lowered the opposition to take into consideration the desire of motherhood in this infective setting. It should be stressed that women with HIV infection, when refused counselling and help, may try for a pregnancy without medical advice with an increase of the risk of sexual and vertical transmission. Women with other transmissible diseases, either infective as hepatitis $\mathrm{C}$ or genetic as cystic fibrosis, receive counselling and are permitted to make their own decisions whether the risk of having an affected child is acceptable to them, even when this risk can be as high as $50 \%$. It seems difficult to deny reproductive counselling and treatment to women infected with HIV as this can reduce the risk of infection for the seronegative partner to zero and reduce tenfold the possibility of transmission to the child..$^{18-21}$

Reproductive counselling to HIV infected women was considered a taboo until recently, but this slight change is due to the improvement of their health conditions and the marked reduction in vertical transmission. Difficulties exist for HIV infected women who cannot conceive spontaneously, as most centres are still reluctant to provide them with full infertility care when required. This discrimination against the reproductive rights of women infected with HIV remains while their chances of having a child are left to the willingness of the infertility specialists to provide reproductive assistance to achieve conception. ${ }^{22}{ }^{23}$

\section{SCENARIO 3}

When both partners are HIV positive and want to have a child, the basic ethical dilemma faced by the clinician remains the same: medical intervention may put a new life at risk of infection with HIV. The setting in which the decision to provide assistance takes place is, however, different, as the possibility that both mother and father may die of their infection should be taken into account, with the child growing up without a parent. We present a couple whom we saw in the early 1990s and discuss how, owing to the improved prognosis for mother and baby, we believe our refusal to treat them is no longer appropriate..$^{24}$

In this case, the couple had presented with secondary infertility, both having had children with previous partners. They had both tested positive for antibodies to HIV-1 in 1989 and the infection had probably occurred through heterosexual transmission. Serum P24 antigen was not found in either of them, the T4 lymphocyte number was low in the man and at the lower limit of normal in the woman. Investigations had also revealed that polycystic ovarian disease and oligospermia were the cause for their infertility. From an infertility viewpoint, the treatment option then included in vitro fertilisation. However in view of their HIV status, after considering all possible implications, they were refused any active treatment for their secondary infertility. This decision was based on the poor prognosis (morbidity and mortality) for HIV patients and the high risk of vertical transmission (14-25\%) in early 1990s.

\section{Discussion}

In the past 10 years, significant advances have been achieved in controlling HIV infection and preventing vertical transmission so as to change the reproductive prospects of infected individuals. ${ }^{45}$ Men and women with HIV infection should be informed of the specific measures to be adopted when they want to have a child. The rate of infertility in these couples may be higher owing to their previous or present life style, less efficient response to infection, and the side effects of psychoactive drugs or antiretroviral compounds. The progress in the efficacy of antiretroviral treatment in controlling the disease and the availability of specific measures (semen washing, elective abdominal delivery) to limit the risk of sexual and materno-fetal infection ${ }^{16} 172526$ has been reflected in the response of some gynaecologists attending to the reproductive desires of these couples. However, this change of attitude has been slow and controversial. The couple presented above would probably have received proper counselling and care if they had achieved a pregnancy on their own, but were denied treatment when the doctors had to take part in achieving fertilisation. In essence, they were refused treatment not because of infection but because of their infertility problem. This seems a controversial decision making process, but fully reflects the difficulties both these couples and their doctors face when decisions entail potential risks for healthy individuals. The "do no harm" comes into question when doctors have to take an active part in the treatment and thus need to take their share of risk and responsibilities. The duty of the medical practitioner is not only to provide appropriate counselling and optimal care but also to provide protection from the known risks.

\section{CONCLUSION}

HIV is now classified as a chronic disease in the Western world because of a significant increase in the quality of life and expectancy with the introduction of highly active antiretroviral therapy. This, along with the developments in reducing vertical transmission and partner infection, has

Debate 
Debate

made it necessary to openly re-discuss the reproductive desires and rights of HIV infected individuals so as to provide the doctors involved in their care with recommendations.

We also know that $75 \%$ of those infected with HIV are in their childbearing years and therefore it is important that this issue is addressed. Our centre is conducting a questionnaire survey of fertility and genitourinary clinics to assess the fertility options currently available to these couples nationwide (Gilling-Smith C and Frodsham L, personal communication). We believe that an open discussion and availability of guidelines will enable the medical practitioner to deal with the reproductive options of these couples in a scientific and ethically appropriate manner.

To summarise, the above recommendations seems to us to fulfil the four underlying ethical principles:

\section{(i) Primum non nocere: first of all, do no harm}

The interventions reduce the existing risks of the mother and the fetus to a very low level. So far, no follow up data have suggested that any of these interventions will cause harm.

\section{(ii) Beneficence: do good}

The couples desperately want to have healthy children. In offering these interventions the chances that they can achieve this is increased.

(iii) Autonomy: respect the individual The couples' informed choice should be respected in the presence of treatment success. Why should these couples be denied treatment in 2003?

\section{(iv) Justice: honest and fair care}

Denial of any infertility treatment for HIV couples now would be inappropriate as the above principles can be applied to the child also.

\section{REFERENCES}

1 Selwyn PA, Antoniello P. Reproductive decision-making among women with HIV infection. In: Johnson MA, Johnstone FD, eds. HIV infection in women. Edinburgh: Churchill Livingstone, 1993.

2 Rudge CJ. Organ donation. Ethical aspects. In: Forsythe JR, ed. Transplant Surg 1997: 1-17.

3 Gilling-Smith C, Almeida P, and BFS Practice and Policy Committee. HIV, hepatitis B and C and infertility: BFS Practice and Policy. Human Fertility 2003; (in press).

4 Lyall EG, Blott $M$, de Ruiter $A$, et al. Guidelines for the management of HIV infection in pregnant women and the prevention of mother-to-child transmission. HIV Med $2001 ; 2: 314-34$

5 Saracco A, Musicco M, Nicolosi A, et al. Man-to-woman sexual transmission of HIV: longitudinal study of 343 steady partners of infected men. J Acquir Immune Defic Syndr 1993;6:497-502.

6 Mastro TD, de V I. Probabilities of sexual HIVtransmission. AIDS 1996;10 (Suppl A):S75-82.
7 Mandelbrot L, Heard I, Henrion-Geant E, et al. Natural conception in HIV-negative women with HIV-infected partners. Lancet 1997;349:850-1

8 Semprini AE, Levi-Setti $P$, Bozzo $M$, et al. Insemination of HIV-negative women with processed semen of HIV-positive partners, Lancet 1992:340:1317-19.

9 Semprini AE, Fiore S, Pardi G. Reproductive counselling for HIV-discordant couples [letter]. Lancet 1997;349:1401-2.

10 Kim LU, Johnson MR, Barton S, et al. Evaluation of sperm washing as a potential method of reducing HIV transmission in HIV-discordant couples wishing to have children. AIDS 1999;13:645-51.

11 Muller $\mathrm{CH}$, Coombs RW, Krieger JN. Effects of clinical stage and immunological status on semen analysis results in human immunodeficiency virus type 1-seropositive men. Andrologia 1998;30(Suppl 1):15-22.

12 Laudat A, Blum L, Guechot J, et al. Changes in systemic gonadal and adrenal steroids in asymptomatic human immunodeficiency virus-infected men: relationship with the CD4 cell counts. Eur J Endocrinol 1995;133:418-24

13 Pena JE, Klein J, Thornton M, et al. Successive pregnancies with delivery of two healthy infants in a couple who was discordant for human immunodeficiency virus infection. Fertil Steril 2002;78:421-3.

14 Marina S, Marina F, Alcolea R, et al. Pregnancy following intracytoplasmic sperm injection from an HIV-1-seropositive man. Hum Reprod 1998;13:3247-9.

15 Panel on Clinical Practices for Treatment of HIV. Guidelines for using antiretroviral agents among HIV-infected adults and adolescents. Recommendations of the Panel on Clinical Practices for Treatment of HIV. MMWR Morb Mortal Wkly Rep 2002;51 (RR-7): 1-55

16 The International Perinatal HIV Group. The mode of delivery and the risk of vertical transmission of human immunodeficiency virus type 1-a meta-analysis of 15 prospective cohort studies. N Engl J Med 1999:340:977-87.

17 Connor EM, Sperling RS, Gelber R, et al. Reduction of maternal-infant transmission of human immunodeficiency virus type 1 with zidovudine treatment. Pediatric AIDS Clinical Trials Group Protocol 076 Study Group. N Engl J Med 1994;331:1173-80.

18 Ethics Committee of the American Society for Reproductive Medicine. Human immunodeficiency virus and infertility treatment. Fertil Steril 2002;77:218-22.

19 Minkoff $\mathbf{H}$, Santoro N. Ethical considerations in the treatment of infertility in women with human immunodeficiency virus infection. $N$ Engl J Med 2000;342:1748-50

20 Gilling-Smith C, Smith JR, Semprini AE. HIV and infertility: time to treat. There's no justification for denying treatment to parents who are HIV positive. BM 2001;322:566-7.

21 Drapkin LA, Anderson J. Human immunodeficiency virus and assisted reproduction: reconsidering evidence, reframing ethics. Fertil Steril 2001;75:843-58.

22 Apoola A, tenHof J, Allan PS. Access to infertility investigations and treatment in couples infected with HIV questionnaire survey. BM 2001;323:1285.

23 Balet $\mathbf{R}$, Lower AM, Wilson C, et al. Attitudes towards routine human immunodeficiency virus (HIV) screening and fertility treatment in HIV positive patients-a UK survey. Hum Reprod 1998;13:1085-7.

24 Smith JR, Forster GE, Kitchen VS, et al. Infertility management in HIV positive couples: a dilemma. BM 1991; 302:1447-50.

25 Nduati $\mathbf{R}$, John $G$, Mbori-ngacha $D$, et al. Effect of breastfeeding and formula feeding on transmission of HIV-1. A randomized clinical trial. JAMA 2000;283:1167-74

26 Anon. Ongoing nucleoside safety review of HIV-exposed children in US studies. 2nd conference on Global Strategies for prevention of HIV transmission from Mother to infants, Montreal, Sept, 1999. 